MATEC Web of Conferences 28, 02002 (2015)

DOI: $10.1051 /$ matecconf $/ 20152802002$

(C) Owned by the authors, published by EDP Sciences, 2015

\title{
Effect of Number of Blades on Performance of Ceiling Fans
}

\author{
Ehsan Adeeb ${ }^{1, a}$, Adnan Maqsood ${ }^{1, b}$ and Ammar Mushtaq ${ }^{1, c}$ \\ ${ }^{1}$ Research Centre for Modeling \& Simulation, \\ National University of Sciences \& Technology, Islamabad, Pakistan 44000
}

\begin{abstract}
In this paper, the effect of number of blades on ceiling fan performance is discussed. This approach helps to satisfy tradeoff between high air flow (performance) and power consumption (energy efficiency). Specifically, variation from two to six blades is considered with nonlinear forward sweep profile. Reynolds Averaged Navier-Stokes (RANS) technique is used to model the flow field induced by the ceiling fan inside a generic room. The performance is gauged through response parameters namely volumetric flow rate, mass flow rate, torque and energy efficiency. The results indicate that mass and volumetric flow rates are maximized for six blade configuration and energy efficiency is maximized for two blade configuration. The study indicates the importance of tradeoff between high air flow through ceiling fan and associated energy efficiency.
\end{abstract}

\section{Introduction}

Ceiling fans are frequently used as a common household appliance in the tropical region. The wide-spread utility of ceiling fans is attributed to affordable purchasing, operational and maintenance costs. Air circulation inside the room and energy consumption are two extremes of design specifications that can be traded off by improving motor and blade designs. Improvement in motor design is generally achieved through material changes that directly results in cost increase. Any alteration in blade design requires manufacturing of new jigs and fixtures in the process line. To satisfy varying costumer requirements, an easy and cost effective solution is to vary the number of blades against the specified performance requirements.

Parker et al. [1] improved the velocity, distribution of air flow and acoustic signature of a three bladed ceiling fan. Numerical results reveal that the tapered design indicates a two fold increase in energy efficiency compared to a conventional blade. Schiavon \& Melikov [2], Bassiouny \& Korah [3] and Mahlia et al. [4] carried out computational as well as experimental studies to improve the air circulation of ceiling fans. It is revealed that the air circulation depends upon fan speed, diameter, location, blade angle and number of blades. Downward velocity of ceiling fan is explicitly dependent on ceiling fan rotation speed. Mahlia et al. [4] proposed a blade profile similar to cambered airfoil. At low rpm, the cambered blade produce less airflow in comparison to conventional fans but at medium and high rpm cambered fan blade shows greater value of airflow. However, the new blades possess higher size to weight ratio and have high level of complexity while manufacturing. Lin and Hsieh [5] enhanced the performance of the hidden ceiling fan using computational modeling and experimental validation. It is found that 'inhale return' phenomena occurs due to inappropriate housing designs. Afaq et al. [6] computationally modeled the flow field of ceiling fan inside a generic square room and conducted parametric study of rake angle for the purpose of design improvement. The results indicate that the fan efficiency can be increased by varying the rake angle. Adeeb et al. [7] studied the effect of nonlinear swept ceiling fan blades on rated air delivery and optimized the design using response surface methods. Specifically sixteen experiments are designed using $2^{\mathrm{k}}$ factorial model. It is found that tip angle of attack and root angle of attack show maximum sensitivity towards rated air delivery. Forward sweep has moderate effect on rated air delivery whereas tip width doesn't have a significant effect. Numerous analytical, computational and experimental techniques have been used by Jain et al. [8], Chiang et al. [9], Lin and Hsieh [10], Rizk et al. [11], Momoi et al. [12] , Prabhakaran and Kumar [13], Sathaye et al. [14], Lubliner et al. [15], Idahosa et al. [16], Kim and Ahn [17] and Makhoul et al. [18] to improve airflow and energy efficiency of ceiling fans. In this study, a profound effort is made to evaluate the effect of number of blades on flow field inside a generic room using Computational Fluid Dynamics (CFD) techniques. The objective of the

\footnotetext{
${ }^{\mathrm{b}}$ Corresponding author: $\underline{\text { adnan@ } @ \text { rcms.nust.edu.pk }}$
} 
research is to interpret the flow field and velocity profile of the fan blade through computational modeling and parametric study. A non-linear blade design and room dimensions are selected from Adeeb et al. [7]. The details of geometric descrition of room and blade profile are discussed first. The computational setup is followed by results, discussion and conclusions.

\section{Description of the Geometry}

The room dimensions and blade profile used in this research are adopted from Adeeb et al. [7]. The height of the room is $4 \mathrm{~m}$ whereas its length and width are $4.5 \mathrm{~m}$ each. Computational model of room can be seen in Fig. 1 where fan disk and room are connected by frozen rotor interface. Gambit ${ }^{\circledR}$ software is used for constructing the geometry and grid generation [21]. The selection of the blade profile is based on its good energy efficiency and volumetric flow rate compared to the other design alternatives considered [7]. The length of the blade is $0.568 \mathrm{~m}$, root and tip angle of attack of $12 \mathrm{deg}$. The detailed geometry of the blade is shown in Fig. 2.

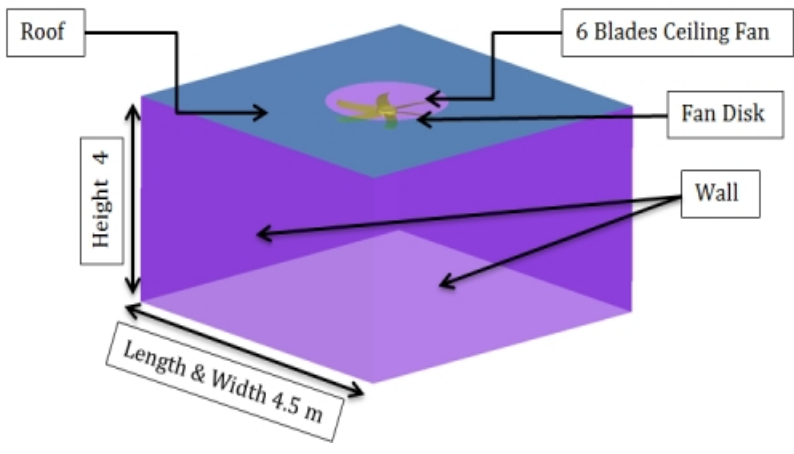

Figure 1. Computational model of room

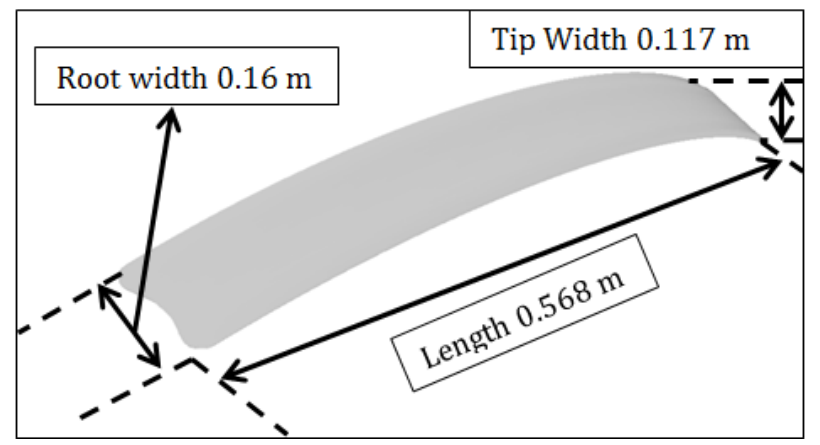

Figure 2. Computational model of fan blade

In this research, numbers of blades are varied from two to six. The angle between two consecutive ceiling fan blades in two, three, four, five and six blade configuration is $180^{\circ}, 120^{\circ}, 90^{\circ}, 72^{\circ}$ and $60^{\circ}$ respectively as shown in Fig. 3.

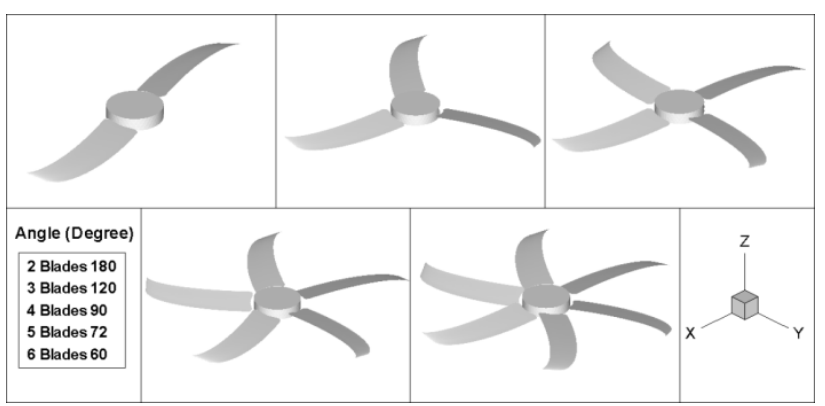

Figure 3. Ceiling fans with varying number of blades

\section{Computational Modeling \& Setup}

Reynolds-Averaged-Navier-Stokes (RANS) system of equations is solved using coupled implicit formulation in Fluent ${ }^{\circledR}[19,20]$. Unstructured tetrahedral scheme is used to mesh the domain. A grid independence study for fan and room is done before deciding the mesh size. The main purpose of mesh study is that by the changing the grid size there is no effect on flow profile and it captures the flow features accurately. Matching grid has been made at interface of room and fan to avoid from discontinuity in flow. Boundary conditions used in this research are listed in the Table 1 . The validation of the numerical data with experimental results is already demonstrated in [7] and not reproduced here for brevity.

Table 1. Boundary Conditions used in fan simulation

\begin{tabular}{|c|c|}
\hline Parameters & Type \\
\hline $\begin{array}{c}\text { Blade, hub and room } \\
\text { boundary condition }\end{array}$ & Wall (no slip) \\
\hline Pressure discretization & Standard \\
\hline Pressure-velocity coupling & SIMPLE \\
\hline Viscous model & SA Model [23, 24] \\
\hline Fan and Room disk & Interface \\
\hline Momentum discretization & First Order Upwind \\
\hline Gradient & $\begin{array}{c}\text { Least Square Cell } \\
\text { Based }\end{array}$ \\
\hline Solver & $\begin{array}{c}\text { 3D Steady, } \\
\text { Incompressible }\end{array}$ \\
\hline
\end{tabular}

\section{Results \& Discussion}

Response parameters to assess the effect of number of blades used in this study are volumetric flow rate, mass flow rate, torque, and energy efficiency. All values of response parameters are captured on a surface of $1.5 \mathrm{~m}$ below the ceiling fan and results are extracted using Tecplot360® [22].

Volumetric flow rate is the volume of air coming down on a specified surface per unit time. Volumetric flow rate for different number of blades is given in Fig. 4. The maximum value of volumetric flow rate is observed for six blade fan and minimum value for fan with two blades. In Fig. 4, an increasing trend in volumetric flow rate is seen by increasing the number of blades. However, it can be seen that the difference between two and three blade configurations is maximum. On the other hand, the 
difference between four and five blade configuration is minimum.

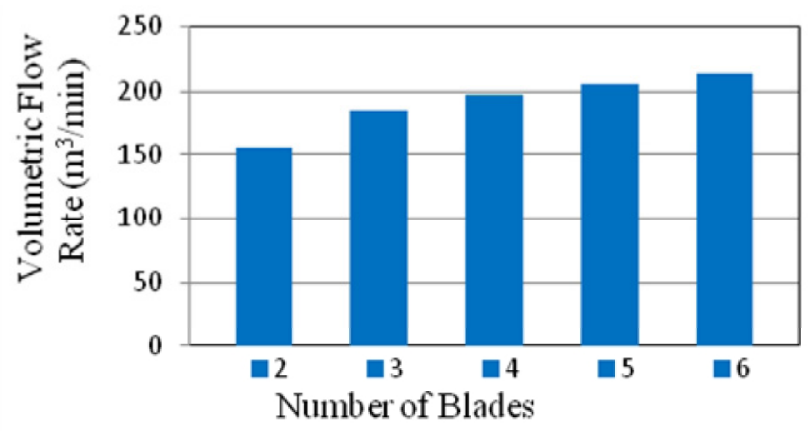

Figure 4. Volumetric flow rate

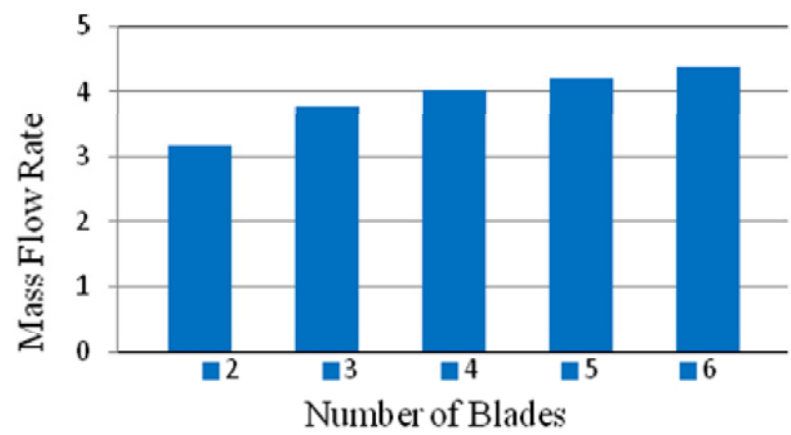

Figure 5. Mass flow rate

Mass flow rate is the mass of a substance that passes through a given surface per unit of time and is expressed as:

$$
m=\rho v \cdot A
$$

where $\mathrm{m}$ is the mass flow rate, $\rho$ is the density, $\mathrm{v}$ is the average velocity and $\mathrm{A}$ is the cross sectional area. Different cases are simulated for ceiling fan having different number of blades and result of mass flow rate is shown in the Fig. 5. An upward trend is also seen by increasing the number of blades. The maximum value is shown by the fan with six blades and minimum value is shown by a fan with two blades. Two blade fan demonstrates minimum value of torque, $1.25 \mathrm{~N} . \mathrm{m}$, as the fan motor require less power. However, a proportionate rise in torque is observed with the more number of blades. For instance, the six blade fan shows maximum value of torque, 3.06 N.m. (Fig. 6).

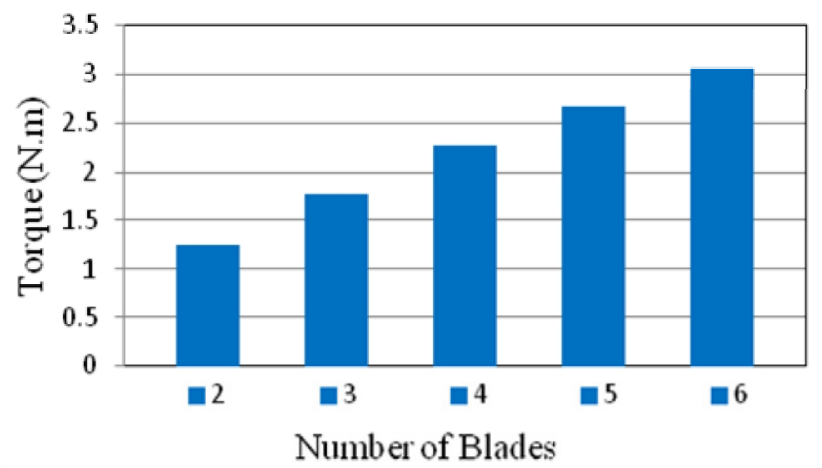

Figure 6. Torque

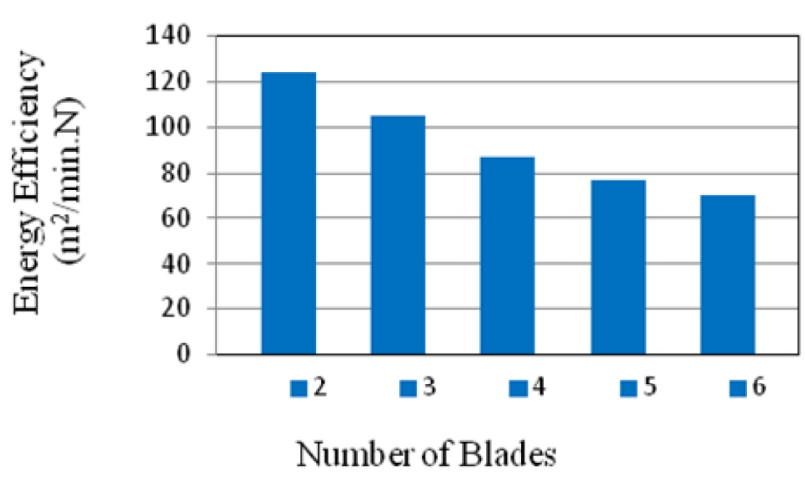

Figure 7. Energy efficiency

Energy efficiency is an important indicator of ceiling fan performance and it is the ratio of volumetric flow rate to torque of fan. Fan with two blades shows maximum value of energy efficiency because its torque value is very small. Minimum value is shown by the fan having six blades because a greater value of mechanical power is required to rotate ceiling fan blade (Fig. 7).
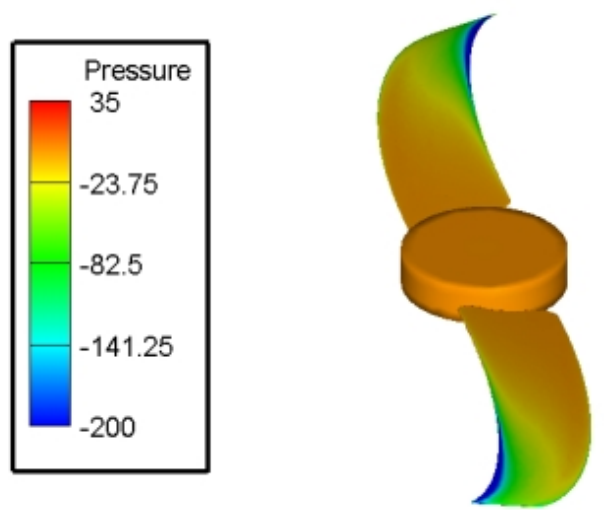

Figure 8. Two Blades pressure contour (upper side)

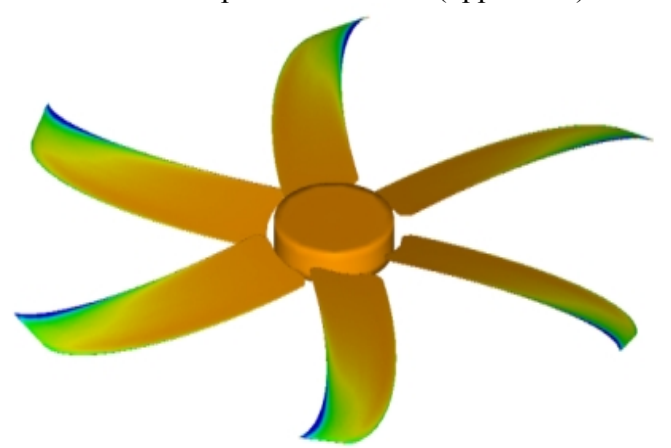

Figure 9. Six Blades pressure contour (upper side)

The pressure contour of fan having two blades and six blades can be seen in Fig. 8-9 and velocity magnitude of fan having two blades and six blades can be seen in Fig. 10-11. The static pressure contours are almost same that demonstrates minimum interference effects. Moreover, the leading edge of the blades show low value of pressure demonstrating a swirl effect from the tips of the blades. This observation is consistent for both two and six blade configurations. From velocity contours, minimal effect of flow field interaction on with the variation in number of blades is inferred. 

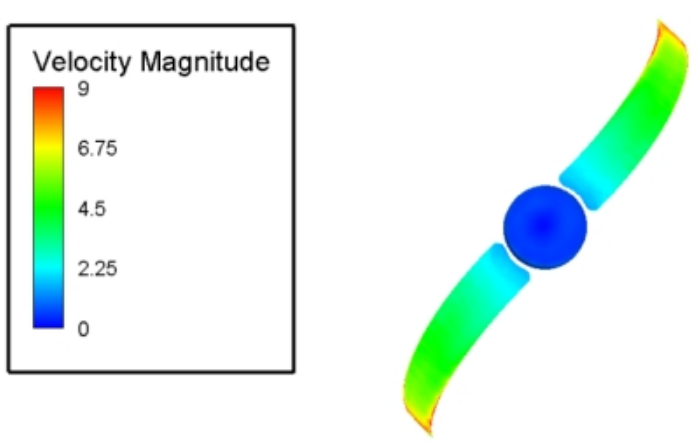

Figure 10. Two blades velocity contour (lower side)

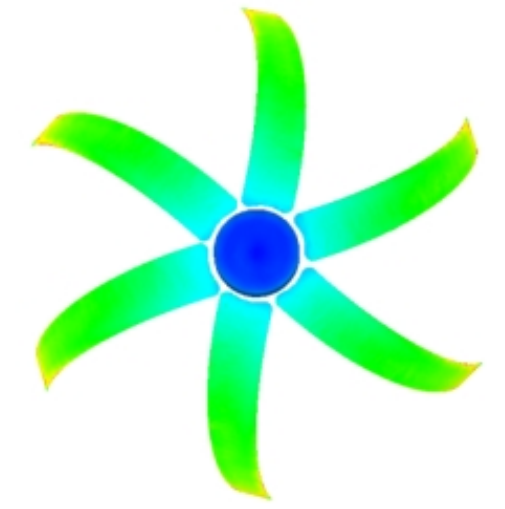

Figure 11. Six blades velocity contour (lower side)

\section{Conclusion}

In this work, a parametric study is carried out to find the effect of number of blades on the performance of ceiling fans. The ceiling fan blade has a peculiar nonlinear forward sweep that helps to improve the air circulation in the room. The number of blades varied are from two to six. It is observed that increasing number of blades results in higher volumetric and mass flow rates. Moreover, minimum interference effects between blades are found for two as well as six blade configurations. If energy efficiency coupled with volumetric flow rate is taken under consideration, a three or four blade configuration is more desirable.

\section{Acknowledgement}

This work is supported by Higher Education Commission (HEC) of Pakistan grant no PDIPFP/HRD/HEC/2013/1943 and Fan Development Institute (FDI), Gujrat, a subsidiary of Pakistan Electric Fan Manufacturing Association (PEFMA), Pakistan.

\section{References}

1. Danny, S.P., Michael P.C, Jeffery K.S, Guan H. Su, Bart D. Hibbs, Development of a High Efficiency Ceiling Fan, in Improving Building Systems in Hot and Humid Climates. 2000: San Antonio.

2. Schiavon, S. and A. K.Melikov, Energy saving and improved comfort by increased air movement. . Energy and buildings 2008.
3. Ramadan, B. and S.K. Nadar, Studying the Features of Air Flow Induced by a Room Ceiling-Fan. Energy and Buildings, 2011. 43.

4. T.M.I. Mahila, Therotical and experimental investigation of energy efficient improvement of the ceiling fan by using aerodynamic blade profile, Journal of energy and environment. 2013

5. Sheam chyun lin and Ming yuan hsieh, An integrated numerical and experimental analysis for enhancing the performance of the hidden ceiling fan, Advances in Mechanical Engineering, 2014

6. Aaqib, A.M., Maqsood, A., Khalid Parvez, Mushtaq, A., Aerodynamic Design and Optimization of an Energy Efficient Ceiling Fan, in $11^{\text {th }}$ IBCAST, Research Center for Modeling and Simulation. 2014, National University of Sciences and Technology: Islamabad.

7. Adeeb, E., Maqsood, A., Mushtaq, A., Zamir, H., Shape Optimization of Non-Linear Swept Ceiling Fan Blades through RANS Simulations and Response Surface Methods, in $12^{\text {th }}$ IBCAST, Research Center for Modeling and Simulation. 2015, National University of Sciences and Technology: Islamabad.

8. Ankur, J., Upadhyay, R.R., Chandra, S., Sainin, M., Kale, S., Experimental Investigation of the Flow Field of a Ceiling Fan. ASME Heat Transfer/Fluid Engineering, 2004.

9. Hsu-Cheng Chiang, C.-s.P., Hsi-Sheng Wu, BingChwen Yang, Measurement of Flow Characteristics of a Ceiling Fan with Varying Rotational Speed in Proceedings of Clima 2007 WellBeing Indoors. 2007.

10. Ming-Yuan Hsieh, S.-C.L.a., An Integrated Numerical and Experimental Analysis for Enhancing the Performance of the Hidden Ceiling Fan. Hindawi 2014.

11. A. Rizk, A.A., N. Guirguis, Simulation comparison between natural and hybrid ventilation by fans at night time for severe hot climate.

12. Y. Momoi, K.S., T. Yamanaka and H. Kotani, Modeling of Ceiling Fan Based on Velocity Measurement for CFD Simulation of Airflow in Large Room.

13. Kumar, S.P.a.M.S., development of glass fiber reinforced polymer composite ceiling fan blade. international journal of engineering research and development. 2(3): p. 59-64.

14. Nakul Sathaye, A.P., Nihar Shah, Virginie Letschert, Potential Global Benefits of Improved Ceiling Fan Energy Efficiency, in ernest orlando lawrence berkeley national laboratory. 2012.

15. M. Lubliner;J. Douglass, P.D.P.a.D.C., PE, performance and applications of gossamer wind ${ }^{\mathrm{TM}}$ solar powered ceiling fans.

16. Idahosa, U, V. V.Golubev, and V.O. Balabanov, An automated optimal design of a fan blade using an integrated $\mathrm{CFD} / \mathrm{MDO}$ computer environment. Engineering applications of computational fluid mechanics. Vol 2, No 2, 2008.

17. Ahn, C.-S. and K.-Y. Kim, Aerodynamic design optimization of a compressor rotor with navier stoke analysis. . 2003. 
18. A.Makhoul, K. Ghali, and N. Ghaddar, Desk fan for the control of the convection flow around occupants using ceiling mounted personalized ventilation. Buildings and environment, 2013.

19. Computational Fluid Dynamics Software Package, Fluent 6.2.16. 2006: New Hampshire.

20. Computational Fluid Dynamics Software Package User Guide,Fluent Ver. 6.2.16. 2006

21. Geometry and Mesh Generation Software Package, Gambit Ver. 2.3.16. 2006.
22. Tecplot360 software package 11.0-1-125 2006. 2006.

23. Zhai, Z., et al., Evaluation of Various Turbulence Models in Predicting Airflow and Turbulence in Enclosed Environments by CFD: Part-1: Summary of Prevent Turbulence Models. 2007.

24. Spalart, P.R.a.A., S. R., A One-Equation Turbulence Model for Aerodynamic Flows, in AIAA. 1992, AIAA. 